\title{
Télescope
}

Revue d'analyse comparée en administration publique

\section{La gouvernance multiniveau et les politiques publiques au sein des municipalités du Canada : reddition de comptes et efficacité}

\section{Robert Young}

Volume 19, numéro 1, hiver 2013

La gouvernance multiniveau

URI : https://id.erudit.org/iderudit/1017150ar

DOI : https://doi.org/10.7202/1017150ar

Aller au sommaire du numéro

Éditeur(s)

L’Observatoire de l'administration publique

ISSN

1929-3348 (numérique)

Découvrir la revue

Citer cet article

Young, R. (2013). La gouvernance multiniveau et les politiques publiques au sein des municipalités du Canada : reddition de comptes et efficacité. Télescope 19(1), 25-42. https://doi.org/10.7202/1017150ar
Résumé de l'article

Cet article fait état des principaux résultats d'un projet de recherche entrepris par un vaste groupe d'universitaires au cours des sept dernières années. L'équipe a centré ses travaux sur la manière dont les négociations intergouvernementales produisent des politiques dans les municipalités canadiennes et sur le rôle des « forces sociales » dans le processus d'élaboration des politiques. L'étude s'intéresse en particulier aux administrations municipales, à la fois en tant que participants au cycle des relations intergouvernementales et en tant que pôles d'échanges des réseaux locaux des forces sociales. De façon générale, les fruits de cette recherche dressent un tableau détaillé de la gouvernance multiniveau au Canada. Les résultats publiés ici sont liés aux questions de reddition de comptes et d'efficacité. Après une brève introduction, ces thèmes sont abordés dans l’ordre. En conclusion, il est question de résultats généraux sur la gouvernance multiniveau au Canada et de la posture des administrations municipales dans le système politique canadien. 


\title{
LA GOUVERNANCE MULTINIVEAU ET LES POLITIQUES PUBLIQUES AU SEIN DES MUNICIPALITÉS DU CANADA : REDDITION DE COMPTES ET EFFICACITÉ
}

\author{
Par Robert Young ${ }^{1}$, Professeur au Département de science politique et Titulaire de la \\ Chaire de recherche du Canada en gouvernance multi-paliers, Université de Western \\ Ontario•young@uwo.ca \\ Traduit de l'anglais
}

\begin{abstract}
RÉSUMÉ Cet article fait état des principaux résultats d'un projet de recherche entrepris par un vaste groupe d'universitaires au cours des sept dernières années. L'équipe a centré ses travaux sur la manière dont les négociations intergouvernementales produisent des politiques dans les municipalités canadiennes et sur le rôle des " forces sociales " dans le processus d'élaboration des politiques. L'étude s'intéresse en particulier aux administrations municipales, à la fois en tant que participants au cycle des relations intergouvernementales et en tant que pôles d'échanges des réseaux locaux des forces sociales. De façon générale, les fruits de cette recherche dressent un tableau détaillé de la gouvernance multiniveau au Canada. Les résultats publiés ici sont liés aux questions de reddition de comptes et d'efficacité. Après une brève introduction, ces thèmes sont abordés dans l'ordre. En conclusion, il est question de résultats généraux sur la gouvernance multiniveau au Canada et de la posture des administrations municipales dans le système politique canadien.
\end{abstract}

\begin{abstract}
This article reports some of the principal findings from a research project undertaken by a large group of scholars over the past seven years. Our focus was on how intergovernmental negotiations produce policy in Canadian municipalities, and on the role of 'social forces' in the policy making process. We have been particularly interested in municipal governments, both as participants in intergovernmental relations and as hubs of networks of local social forces. Our results, overall, paint a thorough picture of multilevel governance in Canada. Here, I have selected findings that bear on questions of accountability and effectiveness. After a brief introduction, and an account of the research, these themes are taken up in turn. I conclude with general findings about multilevel governance in Canada and about the position of municipal governments in the Canadian system.
\end{abstract}

Pour citer cet article : Young, R. (2013). « La gouvernance multiniveau et les politiques publiques au sein des municipalités du Canada : reddition de comptes et efficacité », Télescope, vol. 19, n 1, p. 25-42.

\section{- LES ADMINISTRATIONS MUNICIPALES AU CANADA}

T e pouvoir des administrations locales au Canada est relativement faible. L Lorsqu'on les compare à leurs contreparties dans les autres pays avancés, le pouvoir constitutionnel et juridique des municipalités canadiennes demeure limité

L'auteur tient à remercier le Conseil de recherches en sciences humaines du Canada pour son appui et Kelly McCarthy pour son assistance. 
(Young, 2009a). Sur le plan de la constitution, les autorités locales relèvent des gouvernements provinciaux, ce qui explique le cliché voulant qu'elles soient des " créatures des provinces ». Les gouvernements provinciaux les créent, les dotent de lois, prescrivent de nombreuses politiques qu'elles mettent en œuvre et, fréquemment, les éliminent par le procédé de la fusion. Bien sûr, ces mêmes gouvernements provinciaux doivent se montrer sensibles envers l'opinion publique et les pressions locales, tout en se souciant des économies locales. Cela se révèle vrai en particulier dans les cas où une grande ville représente une large portion de la population d'une province. Winnipeg, pour prendre un cas extrême au Canada, regroupe $57 \%$ de la population du Manitoba.

Il n'est pas étonnant de constater que la position financière des municipalités est relativement fragile. Les dépenses des administrations locales représentent $4,1 \%$ du PIB canadien, alors qu'en 2009, la moyenne pour les vingt-neuf pays membres de l'OCDE s'établissait à $12,2 \%{ }^{2}$. Au Canada, la plupart des gouvernements « supérieurs » ne permettent pas aux municipalités d'accéder à des bases de taxation croissant avec l'économie, telles que les taxes de vente et les impôts sur le revenu. Par conséquent, les administrations locales dépendent habituellement de la taxe foncière et des taxes qui y sont liées, ce qui représente $50 \%$ de leurs revenus totaux et $62 \%$ de leurs revenus autonomes (Sancton et Young, 2009, p. 502). Leurs autres revenus proviennent principalement des ventes de biens et services et des transferts, qui sont majoritairement l'affaire des provinces et, pour la plupart, conditionnels.

Dans le monde intergouvernemental canadien, les municipalités offrent une faible concurrence (Breton, 1987, p. 319-322), mais elles disposent de quelques ressources. Elles détiennent beaucoup d'information sur la localité. Les organismes provinciaux et fédéraux disposent également de beaucoup d'information. En Ontario, par exemple, dans le domaine de l'établissement des immigrants, le ministère fédéral, Citoyenneté et Immigration Canada, ainsi que le ministère provincial des Affaires civiques et de l'Immigration possèdent de l'information localisée à propos des mouvements des immigrants, des organismes d'établissement, des besoins linguistiques, etc. Mais ces données ne sont pas intégrées à d'autres connaissances de la municipalité liées à l'établissement des immigrants - comme les questions de logement, les bibliothèques, les loisirs, les entreprises locales, etc. L'information locale intégrée est la spécialité des administrations municipales. De plus, les municipalités jouissent d'un monopole d'expertise dans certains domaines, en particulier dans l'aménagement du territoire et le zonage. De surcroît, elles possèdent de forts liens avec les intérêts organisés à l'échelle locale. Dans la mesure où la gouvernance moderne s'appuie sur des consultations et des collaborations avec les expertises et les ressources des secteurs privé et bénévole, les municipalités ont un avantage sur le plan local comparativement aux autres ordres de gouvernement. Enfin, presque tous les gouvernements provinciaux ont légiféré dans l'optique d'augmenter l'autonomie des municipalités, en particulier celle des grandes villes, souvent en remplaçant des pouvoirs étroitement définis par des sphères plus vastes de responsabilité (Garcea et LeSage, 2005).

2 Calcul effectué à partir des données de l'OCDE, 2012, tableau 2. 
Au cours des quinze dernières années, on a assisté à une agitation considérable au sujet de la structure et du fonctionnement des administrations municipales au Canada. Ce phénomène a été stimulé par des facteurs environnementaux profonds (les changements démographiques, les innovations technologiques, les changements dans la culture politique et la restructuration économique occasionnée par une mondialisation plus marquée). La cause immédiate des changements réels dans la posture fédérale face aux municipalités a été le lobbying intense pratiqué par les maires des grandes villes et certaines associations d'entreprises. Le gouvernement du Parti libéral du Canada dirigé par le premier ministre Paul Martin (2003-2006) a introduit un "nouveau pacte " pour les villes et les collectivités, qui incluait une pleine remise de la taxe de vente fédérale sur les dépenses des municipalités et des communautés et le transfert vers les municipalités d'une partie de la taxe fédérale sur l'essence; de plus, un ministère d'État pour l'Infrastructure et les Collectivités a été mis sur pied afin de créer un interlocuteur fédéral pour les municipalités, le premier depuis la fin des années 1970 (Berdahl, 2006). Cette avancée politique a été largement annulée sous le leadership du conservateur Stephen Harper (2006-). Les dépenses en infrastructures municipales ont été maintenues (puis augmentées fortement au cours de la crise de 2008-2009), mais la politique de "fédéralisme d'ouverture " des conservateurs implique un plus grand respect des champs de compétence prévus par la constitution, et les municipalités canadiennes relèvent de l'autorité des provinces (Young, 2006 et 2011). L'intensité des relations entre le fédéral et les municipalités s'est donc globalement atténuée.

Dans les prochaines pages, nous présentons succinctement nos recherches sur la gouvernance multiniveau au Canada en mettant l'accent sur deux aspects précis : la reddition de comptes et l'efficacité.

\section{- LA RECHERCHE}

C'est en raison de l'intérêt croissant pour les affaires municipales au cœur de l'ordre du jour politique national (Andrew, Graham et Phillips, 2002) qu'une équipe de recherche a été formée afin d'étudier la gouvernance multiniveau et la politique publique dans un contexte municipal. À son pinacle, le groupe était constitué de plus de quatre-vingt-dix chercheurs ainsi que de nombreux assistants de recherche étudiants. Les activités du groupe étaient axées sur les politiques publiques qui existent dans les espaces municipaux, qui ne sont bien évidemment pas toutes générées par les administrations municipales en tant que telles. Les chercheurs comptaient explorer la création de telles politiques et les analyser en tant que fonction des négociations intergouvernementales qui produisent ces politiques et de l'implication (ou non) des forces sociales dans les processus d'élaboration des politiques. Malgré l'importance de ce groupe de recherche, il a été impossible d'explorer tous les domaines de politiques. Six domaines ont donc été sélectionnés : la planification des mesures d'urgence, les immeubles fédéraux, la constitution de l'image sur le plan municipal, l'établissement des immigrants, les infrastructures et les politiques autochtones en milieu urbain. Ces domaines promettaient de révéler des relations entre les municipalités et le fédéral dans 
lesquelles les gouvernements provinciaux servaient d'intermédiaires. Certains aspects comme la visibilité, les principaux instruments d'élaboration des politiques et les champs de compétence varient selon les domaines. De plus, certains secteurs impliquent des services aux citoyens alors que d'autres concernent des questions physiques ${ }^{3}$.

L'équipe a produit des études comparatives dignes de mention portant sur la position des municipalités dans les fédérations et leurs relations avec les gouvernements fédéraux (Lazar et Leuprecht, 2007). Des études traitaient également du contexte de la participation du gouvernement fédéral dans les villes au Canada (Carroll et Graham, 2009). Mais la plupart des recherches étaient orientées vers l'élaboration de politiques dans l'espace municipal canadien et elles avaient été conçues pour cibler ce qui différenciait les provinces, les domaines politiques et les municipalités. Dans chaque province, la plus grande ville était étudiée, avec dans chacun des cas une focalisation sur quatre des six champs de politique (Horak et Young, 2012). Les chercheurs ont également observé deux domaines de politique dans chaque province, en enquêtant sur l'élaboration des politiques au sein de quatre municipalités de taille différente (l'un des aspects inhabituels de ces études réside dans l'intérêt pour le fonctionnement de la gouvernance multiniveau dans les petites villes, les villages, voire les municipalités rurales) (Harvey et Young, 2012; Henstra, à paraître; Ircha et Young, à paraître; Peters, 2011; Tolley et Young, 2011).

L'histoire des études sur le fédéralisme au Canada est à la fois ancienne et remarquable (Simeon, 2002; Gagnon, 2009), mais très peu de liens ont été faits entre d'une part les recherches sur les relations fédérales-provinciales et d'autre part celles sur les administrations municipales. L'équipe de recherche avait donc pour objectif de combler ce vide. En raison de l'état embryonnaire de l'observation de la gouvernance multiniveau au Canada (Young et Leuprecht, 2006), l'enquête n'a pu démarrer avec des hypothèses claires. C'est plutôt une liste de questions qui a guidé la recherche. Du côté intergouvernemental, l'étude s'est d'abord intéressée à la documentation sur les relations entre les municipalités et le fédéral. Il était également prévu d'explorer :

- la manière dont les provinces jouent leur rôle d'intermédiaires dans les relations municipales-fédérales;

- la place des politiciens dans les relations intergouvernementales, par opposition aux hauts fonctionnaires;

- l'effet des différends idéologiques dans l'élaboration des politiques;

- l'effet des différentes ressources dont disposent les divers gouvernements;

- les effets qu'aurait pu avoir la nouvelle gestion publique sur la gouvernance multiniveau;

- comment l'accroissement de la collaboration verticale pourrait stimuler la collaboration horizontale entre les administrations locales.

En matière de gouvernance, certaines questions préoccupaient les enquêtes sur le rôle des forces sociales dans le processus d'élaboration des politiques. L'équipe souhaitait documenter les intérêts impliqués dans l'élaboration des politiques et le

3 Pour plus d'information sur le projet de recherche, voir www.ppm-ppm.ca 
moment de leur intervention - établissement de l'ordre du jour, définition des problèmes, établissement des options de politiques, prise de décision, mise en œuvre. La principale question consistait à déterminer l'influence du milieu des affaires sur la détermination des politiques, une question qui domine l'étude des administrations locales en Amérique du Nord depuis des décennies. Quel pouvoir possède le milieu des affaires sur les politiques qui voient le jour dans les municipalités? Finalement, l'équipe s'est demandé s'il était possible pour le milieu des affaires, ou un autre groupe d'intérêt, de " changer d'échelle " pour agir de manière efficace à des échelons plus élevés de gouvernement.

Au-delà de tout cela, une autre préoccupation était l'évaluation de la qualité des politiques publiques. L'un des objectifs de l'étude était d'évaluer les politiques afin de suggérer des changements dans le processus d'élaboration des politiques ou dans les politiques elles-mêmes avec pour but de les améliorer. Les chercheurs avaient pour mission d'observer de nombreux aspects des politiques - l'actualité, l'échelle, la cohérence, l'originalité, l'efficacité, l'équitabilité et la pertinence de la définition des problèmes. Or au final deux principaux critères ont servi à déterminer la qualité des politiques des municipalités canadiennes : l'efficacité (les résultats obtenus par la politique par rapport à ses objectifs) et la réactivité (la fidélité de la politique aux préférences locales). Dans cet article, nous nous préoccupons principalement de l'efficacité et de la réactivité dans le contexte de l'élaboration des politiques multiniveaux.

\section{- LA REDDITION DE COMPTES DANS LA GOUVERNANCE MULTINIVEAU}

L'un des problèmes bien connus de la gouvernance multiniveau est la difficulté des citoyens de tenir les gouvernements responsables des politiques qu'ils ont mis en œuvre. L'obligation de rendre compte force les gouvernements à la transparence, elle les oblige à se tenir prêts à expliquer ou justifier leurs politiques et à faire face aux mécontentements si le public est insatisfait (Papadopoulos, 2010). De bonnes raisons nous poussent à croire que la gouvernance multiniveau agit au détriment de la reddition de comptes. Cette dernière est difficile à réaliser lorsque les rencontres sont privées et que les citoyens ne sont pas informés des positions initiales des divers gouvernements, des intervenants consultés, des résultats potentiels considérés et de la distribution de la responsabilité de la décision. Ces faiblesses ont été remarquées de manière qualitative (Pierre et Peters, 2005) et on trouve de forts indices quantitatifs montrant que le vote économique - le fait de punir ou de récompenser les élus pour leur performance économique nationale est moins présent dans les fédérations, où l'attribution des responsabilités est plus malaisée que dans les États plus unitaires (Anderson, 2006).

Notre recherche a démontré que l'obligation de rendre compte pouvait se révéler faible dans certains domaines de la politique. Les programmes d'infrastructure en sont le meilleur exemple. Les municipalités proposent des projets, puis un comité fédéral-provincial prend les décisions (au Québec, les groupes sont formellement séparés entre le fédéral et le provincial). Les citoyens ignorent l'ensemble des projets proposés ainsi que les raisons pour lesquelles certains financements sont 
refusés ou approuvés. Peu d'information existe sur le poids décisionnel relatif dont disposent les intervenants provinciaux et fédéraux et, détail révélateur, il n'y a pas la moindre transparence à propos de l'influence des autres sources sur les comités - des lobbies municipaux, des membres du Parlement, des ministres du cabinet ou d'autres intérêts. Les membres et ministres locaux du Parlement annoncent les projets retenus avec tambours et trompettes, mais il n'est pas permis aux citoyens de prendre connaissance de la provenance de la décision d'appuyer un projet plutôt qu'un autre.

Dans d'autres espaces politiques, souffrant moins des complexités de la gouvernance multiniveau, il est possible d'établir une forme de transparence. Dans le domaine de l'établissement des immigrants, par exemple, trois provinces (le Québec, le Manitoba et la Colombie-Britannique) ont fait l'objet de transferts de programmes et de financement du fédéral vers les gouvernements provinciaux, et ce sont ces derniers qui sont tenus responsables des politiques concernées. Pour les autres provinces, le gouvernement fédéral apporte du financement aux organismes qui administrent les programmes d'immigration et il demeure responsable, au bout du compte, du choix des politiques. Dans le domaine de la politique pour les Autochtones vivant en milieu urbain, le gouvernement fédéral possède un contrôle final sur les programmes engendrés par la Stratégie pour les Autochtones vivant en milieu urbain et en est responsable. D'autres programmes sont principalement financés par des gouvernements provinciaux responsables, malgré le fait que les organisations autochtones puissent jouir d'importants pouvoirs de prise de décision. Dans le cadre de leurs activités de constitution de leur image de marque, les municipalités profitent d'une large autonomie et sont conséquemment responsables. La situation est moins claire dans le cas de la planification des mesures d'urgence, en raison de la manière dont sont intégrées les règles et les primes de rendement; le tout forme un système légèrement déjanté, dans lequel la reddition de comptes pour les politiques n'est pas facilement réalisable. En terminant, nos études en matière de dessaisissements montrent que le gouvernement fédéral est clairement responsable de ses décisions initiales. Mais les résultats finaux des politiques sont déterminés par des processus aussi flous que ceux qui règnent dans le monde des infrastructures, impliquant des organismes communautaires, des entreprises privées, des municipalités, de nombreux organismes fédéraux et, joueurs importants, des ministères provinciaux. Globalement, la question demeure controversée au Canada. Dans certains domaines, on constate le manque d'obligation de reddition de comptes qui caractérise les structures et les processus complexes de l'Union européenne, alors que dans d'autres, de moins nombreux organismes et ordres de gouvernement sont impliqués de manière concrète dans la prise de décision et on trouve là une meilleure transparence.

\section{- L'EFFICACITÉ}

La véritable gouvernance multiniveau n'est pas tellement répandue au Canada : il existe quelques rares incursions dans ce territoire, où des représentants des trois ordres de gouvernement délibèrent ensemble sur les politiques, et quelques 
exemples encore moins nombreux incluent des intervenants issus des forces sociales. Les Accords de développement urbain signés par Winnipeg, Vancouver, Saskatoon, Regina et les provinces du Manitoba, de la Colombie-Britannique et de la Saskatchewan représentent la forme la plus pure de gouvernance multiniveau au Canada. Ces accords incarnent une approche de type " tous les gouvernements ", dans le cadre de laquelle tous les intervenants pertinents de tous les ordres ont apporté à la table leurs capacités et leurs ressources pour départager les rôles dans la prise en charge de certains problèmes complexes, par exemple la pauvreté extrême et l'abus de drogues dans le centre-ville est de Vancouver. Ces projets étaient minés par des coûts de fonctionnement élevés, en particulier en raison de l'implication des forces sociales, mais il semble qu'ils se soient avérés relativement efficaces (Bradford, 2007; Leo, 2006). Le gouvernement du Parti conservateur a éradiqué les Accords de développement urbain.

Le tripartisme existe également dans d'autres domaines et programmes politiques au Canada. Il est cependant plus fréquent de trouver des négociations bilatérales multiples dans lesquelles le gouvernement provincial joue souvent un rôle primordial. Comme le disent les analystes de l'élaboration des politiques de Calgary, la gouvernance multiniveau " peut être définie comme une mosaïque complexe de relations bilatérales qui connaît parfois des expansions vers des programmes ou des conflits trilatéraux " (Miller et Smart, 2012, p. 26). Il est également vrai que certaines initiatives politiques substantielles se passent presque entièrement de la consultation des administrations municipales. Dans le domaine de l'établissement des immigrants, par exemple, le gouvernement fédéral et les gouvernements provinciaux dotés de responsabilités dans le domaine négocient directement avec les organismes sans but lucratif qui offrent des cours de langue, du counselling d'emploi, des services de santé spécialisés, etc. Ces relations contractuelles sont complexes, mais les fonctionnaires municipaux n'y sont impliqués que de manière marginale, voire pas le moins du monde. Un système semblable est en place dans la plupart des programmes offerts par les gouvernements des provinces aux Autochtones en milieu urbain.

Néanmoins, il existe aussi des initiatives politiques nécessitant une coordination des activités des trois ordres de gouvernement de manière à atteindre leurs objectifs. Les programmes d'infrastructure en font partie. Ceux-ci ont débuté en 1993, pour ensuite proliférer en un déploiement d'initiatives spécialisées, dont le Plan d'action économique du Canada introduit au cours de la crise de 2008-2009 afin de stimuler l'économie. Selon la formule standard, les coûts des projets sont partagés en parts égales entre les trois ordres de gouvernement. Les municipalités proposent des projets et les négociations fédérales-provinciales (ainsi que l'intervention que parvient à imposer l'administration municipale concernée) déterminent quels projets seront financés ${ }^{4}$. Les municipalités passent ensuite à la mise

4 Dans certaines provinces, les Comités de gestion qui administrent les programmes d'infrastructure ont été conseillés par des comités consultatifs comprenant des représentants des associations municipales. Dans les projets très vastes tels que le réaménagement du canal de Lachine et l'expansion du canal d'évacuation des crues du Manitoba, d'intenses consultations publiques ont été tenues, bien qu'elles n'aient porté que sur la mise en œuvre. 
en œuvre, sous la supervision des autres gouvernements. Voici un cas dans lequel un manque grave de capital d'investissement municipal se raccorde au désir du gouvernement fédéral d'augmenter la demande économique ${ }^{5}$. Les immeubles fédéraux sont un autre secteur où se produisent encore plus interactions. La coordination est nécessaire lorsque le gouvernement fédéral entreprend de nouveaux développements. Cependant, au cours des deux dernières décennies, une plus grande interaction a été engendrée par la politique d'Ottawa de fermeture de ses installations militaires et d'abandon de son contrôle des ports et aéroports. Ces décisions ont entraîné des négociations multiniveaux qui se sont parfois révélées pour le moins intenses. Il existe aussi une interaction dans le domaine de la planification d'urgence. Le Conseil canadien des accidents industriels majeurs, par exemple, comptait des représentants des gouvernements provinciaux et fédéral, de nombreuses municipalités et dix-sept associations industrielles. Cet organisme a cependant été éliminé (Henstra, à paraître). Généralement, ce domaine est caractérisé par des politiques fédérales d'encadrement, des politiques provinciales qui les élaborent et des plans et des activités au niveau municipal qui se conforment, plus ou moins, aux lignes directrices provinciales. La politique en la matière est véritablement " nichée " (Hooghe et Marks, 2003) ${ }^{6}$. Dans le cas des Autochtones en milieu urbain, le gouvernement fédéral a continué de soutenir la Stratégie pour les Autochtones vivant en milieu urbain, laquelle finance les initiatives locales. Les représentants municipaux des villes dans lesquelles la Stratégie est mise en œuvre participent en compagnie des fonctionnaires provinciaux et fédéraux et de représentants de la communauté autochtone à la définition des problèmes et à l'allocation du financement.

Une vision d'ensemble de l'efficacité de la gouvernance multiniveau est sans doute plus facilement envisageable en examinant les obstacles qui lui barrent la route. L'efficacité nécessite la coordination des agendas de trois ordres de gouvernement et leur coopération dans l'injection des ressources (Horak, 2012a). Cela requiert interaction et confiance. L'un des obstacles à l'efficacité est le simple roulement du personnel politique. Les politiciens doivent piloter la formation des nouveaux arrangements intergouvernementaux et doivent se montrer prêts à approuver projets et programmes négociés par leurs fonctionnaires. Mais les élections aux trois paliers de gouvernement, à un rythme différent et représentant parfois d'importants

\footnotetext{
5 Les programmes d'infrastructure ont été introduits par le gouvernement libéral de Jean Chrétien, mais ont été maintenus par le gouvernement conservateur de Stephen Harper. Les infrastructures, de même que l'enseignement supérieur et la santé, ont toujours représenté des exceptions au principe du fédéralisme d'ouverture, selon lequel le gouvernement fédéral respecte les compétences provinciales. En 2010, alors que le programme de dépenses urgentes pour stimuler l'économie s'essoufflait, M. Harper a exprimé sa reconnaissance pour le fait que les gouvernements locaux se soient montrés prêts, volontaires et aptes à proposer des projets viables en peu de temps, de manière à ce que l'argent puisse couler. Comme il l'a déclaré aux représentants municipaux, « il nous est difficile de dépenser de l'argent » (Harper, 2010).

6 II existe également une coopération informelle, élaborée au moyen des programmes de formation fédéraux et des décisions contingentes. Dans la municipalité régionale de Halifax, par exemple, le bureau du coordonnateur des urgences est situé dans le même immeuble que le Bureau provincial de gestion des urgences et le représentant régional de Sécurité publique Canada (Grieve et Turnbull, à paraître).
} 
renouvellements de personnel, signifient que durant de longues périodes les nouvelles administrations sont en phase de formation, sont tournées vers elles-mêmes et prendront du temps avant de faire confiance à leurs contreparties des autres paliers. L'annexe 1 en est l'illustration. On y voit l'histoire électorale de la Ville de Montréal, de la province de Québec et du gouvernement fédéral. Les parties grises représentent des périodes d'un an suivant un changement de gouvernement, que ce soit au niveau fédéral ou provincial. De toute évidence, les conjonctures favorables aux ententes multiniveaux sont limitées, alors qu'un palier ou un autre est en réorganisation plus de $35 \%$ du temps. Si l'on considère que la coopération est également plus difficile au cours de l'année suivant l'élection d'une nouvelle administration municipale, les créneaux s'amenuisent encore plus, tel qu'il est démontré dans l'annexe 2.

Au Canada, en général il n'y a pas de partis politiques à l'échelle municipale (à l'exception des grandes villes du Québec et de la Colombie-Britannique). De plus, on tient habituellement pour acquis qu'il n'existe que peu de liens entre le système fédéral des partis et les formations politiques provinciales (malgré de grands chevauchements sur le plan des étiquettes et des indices croissants à l'effet contraire - Esselment, 2010 et 2011). En matière de gouvernance multiniveau, cette situation entraîne des résultats mitigés. Il y a des circonstances où l'harmonie partisane facilite les ententes, et d'autres où les différends idéologiques entravent la coordination intergouvernementale. Mais le principal problème représenté par les structures de parti demeure leur absence systématique sur la scène municipale. Il a été démontré dans nos études que les voies politiques sont très importantes dans certains champs de politique - en particulier dans le cas des infrastructures et des immeubles fédéraux. Ceci est en partie attribuable à l'absence de canal de communication officiel et reconnu au sein du gouvernement fédéral, par lequel les administrations municipales pourraient faire cheminer leurs demandes. Les contacts politiques cependant demeurent sporadiques et désordonnés. Un maire peut jouir d'une intime relation avec un membre du Parlement fédéral ou avec le ministre régional d'une province, mais ces liens demeurent singuliers et personnels. Lorsque les élus changent, ces relations avantageuses sont brisées. Des positions partisanes bien connues et comprises peuvent structurer les relations multiniveaux. Peu importent les effets sur des politiques particulières, les valeurs partisanes offrent ordre et prévisibilité aux relations intergouvernementales? Celles-ci peuvent s'accomplir au sein du système canadien, mais il faut du temps.

La coopération multiniveau a tendance à s'avérer plus efficace lorsqu'existe un leadership résolu au niveau municipal. Cela est particulièrement remarquable dans l'obtention des approbations de projets d'infrastructure et dans le domaine

7 Toutes les études dont font mention Lazar et Leuprecht (2007) couvraient la politique des relations municipales-fédérales. Dans leur sommaire, Lazar et Leuprecht $(2007$, p. 8) écrivent que « dans tous les cas européens et dans ceux de l'Afrique du Sud et du Mexique, les partis politiques ont un effet d'intégration qui assure que les intérêts municipaux sont compris au niveau national ». Cet effet est engendré par l'accumulation des mandats, la sélection de candidats nationaux parmi les politiciens municipaux et les affinités partisanes. Les pays européens inclus dans l'étude sont l'Espagne, la Suisse, I'Allemagne et la France. 
des immeubles fédéraux. La Ville de Toronto, par exemple, ne possédait pas jadis de stratégie cohérente d'infrastructure, et les projets financés se sont montrés en deçà des attentes, du moins du point de vue de la Ville. Avec le temps, cependant, la demande s'est focalisée autour d'un objectif unique, soit le soutien des autres niveaux de gouvernement pour le transport en commun. Cet objectif a été poursuivi avec détermination et consistance pendant des années, étayé par la communauté des gens d'affaires et d'autres coalitions plus vastes, et la Ville a connu un franc succès (Horak, 2012a, p. 234-238). De manière similaire, les municipalités doivent se doter de politiques cohérentes à l'interne. Les plus grandes villes profitent d'unités spécialisées dédiées aux relations intergouvernementales, alors que l'efficacité des plus petites municipalités dépend largement du leadership des maires. Les maires au Canada ne sont pas des maires forts au sens américain du terme, mais ils possèdent une autorité reconnue dans la représentation de leur municipalité à l'externe face aux autres ordres de gouvernement.

Un autre obstacle se dressant sur la route d'une gouvernance multiniveau efficace demeure la propension du gouvernement fédéral à agir de manière unilatérale. Tel qu'il a été noté précédemment, Ottawa ne possède pas d'institution centrale qui lui permet de se lier au secteur municipal dans son ensemble, malgré les efforts de longue date de la Fédération canadienne des municipalités et son quasisuccès dans sa tentative de construire des institutions consultatives avec le gouvernement Martin (Chenier, 2009). Les ministères pris isolément sont en mesure de discuter de politiques et de programmes, mais ils n'hésitent pas à prendre des initiatives politiques sans consulter les municipalités concernées. Ce phénomène est particulièrement frappant dans le domaine des immeubles fédéraux, où dessaisissements et fermetures ont été légion. La fermeture annoncée d'une installation place la communauté locale en état de choc, les politiciens et les gens d'affaires mobilisent de grandes coalitions pour protester, des pressions sont effectuées contre des membres du Parlement, le gouvernement provincial est conscrit; bref, c'est l'agitation politique. Soit la décision est infirmée soit, plus fréquemment, les intérêts locaux élaborent un plan et le gouvernement fédéral se retrouve à fournir une aide de transition et des mesures correctives. De toute façon, les politiques sont gravement dysfonctionnelles et les transitions économiques difficiles. Une approche consultative de la gouvernance multiniveau serait plus efficace (Ircha et Young, à paraître).

Occasionnellement, la gouvernance multiniveau produit des pièges de décision conjointe. Le nombre d'intervenants et leurs ordres du jour presque irréconciliables empêchent parfois tout progrès. Les impasses entraînent généralement des gestes unilatéraux de la part du fédéral ou du provincial, mais lorsque toutes les parties impliquées disposent de ressources importantes et souffrent d'objectifs conflictuels, un blocage peut survenir. Encore une fois, les exemples sont fréquents dans le domaine des immeubles fédéraux, où les plans de rénovation souffrent souvent des mésententes. Par exemple, un parc urbain qui devait être construit sur le site d'une ancienne base aérienne à Downsview (Ontario) demeure bloqué depuis quinze ans (Horak, 2012a, p. 238-241). La réfection des quais de Toronto a donné lieu à des conflits et des verrouillages, à des plans embourbés, détournés, 
puis bloqués durant des décennies en raison d'objectifs changeants et incompatibles et à des interventions politiques tranchantes (Sanderson et Filion, à paraître). En revanche, certains projets de réaménagement complexes impliquant les trois ordres de gouvernement se sont déroulés relativement sans anicroche : le projet du canal de Lachine à Montréal en est un bon exemple (Bherer et Hamel, 2012, p. 125-129).

Dans le cas du canal de Lachine, les relations entre les trois paliers de gouvernement ont été institutionnalisées sous la forme d'un organisme, le Pôle des Rapides, ainsi que dans un ensemble de comités qui impliquaient à la fois des intervenants gouvernementaux et non gouvernementaux. L'institutionnalisation est un outil qui permet de formaliser les relations intergouvernementales, tout en les protégeant quelque peu des pressions politiques transitoires. La revitalisation du secteur riverain des quais de Toronto a été empêtrée pendant des dizaines d'années par la concurrence et les conflits entre les organismes créés par les trois paliers de gouvernement. Au final, cependant, avec la mise sur pied de l'organisme Waterfront Toronto - doté d'un conseil d'administration auquel chaque ordre de gouvernement nomme quatre membres -, on a vu apparaître un véhicule permettant la coordination de la planification du développement. Un cas similaire est celui du COVAN, le Comité d'organisation des Jeux olympiques et paralympiques d'hiver de 2010 à Vancouver. Ce groupe puissant incluait des représentants municipaux, provinciaux et fédéraux, des personnes nommées par les Comités olympique et paralympique canadiens, ainsi qu'un représentant des Premières Nations. L'organisme s'est montré très efficace dans la planification et la mise sur pied d'événements d'une très haute complexité (Hutton, 2012).

La concurrence intermunicipale - ou " horizontale " - nuit à l'élaboration efficace des politiques multiniveaux. Selon le point de vue de certains analystes, l'augmentation des collaborations verticales entraîne une plus grande coopération horizontale (Agranoff et McGuire, 2003). Nous n'avons pu déceler de données corroborant cette hypothèse dans les domaines de politique qui ont fait l'objet de nos observations. Peu de coopération a été constatée entre les municipalités en ce qui a trait à l'établissement des immigrants et aux politiques pour les Autochtones vivant en milieu urbain. On a pu noter des traces de collaboration dans le domaine des infrastructures, où les applications conjointes pour des installations récréatives et d'autres biens publics renforçaient la cause des communautés plus petites. La coopération dans la planification des urgences s'est produite lorsque la régionalisation a été encouragée par les gouvernements provinciaux, tel que ce fut le cas en Nouvelle-Écosse ainsi qu'à Terre-Neuve-et-Labrador. Dans le domaine de la construction des images de marque, on a principalement constaté une véritable concurrence. La majorité des coopérations intermunicipales ont été relevées dans le domaine des immeubles de propriété fédérale, en raison de dessaisissements par le gouvernement fédéral d'installations aux implications réellement régionales, comme les aéroports et les grandes bases militaires.

Dans l'ensemble, la gouvernance multiniveau au Canada est efficace lorsque l'on y a recours. Certains cas de coûts de revient élevés, de mésententes et de délais très longs ont été remarqués, mais on assiste à de nombreux exemples où les 
trois ordres de gouvernement ont réussi à coordonner leurs expertises et leurs ressources à la poursuite d'un objectif qui était prioritaire pour chacun d'entre eux. La majeure partie des créations et des mises en œuvre des politiques en action dans les municipalités est l'affaire de gouvernements opérant seuls; d'autres politiques sont mises en fonction de manière bilatérale, habituellement par le truchement de coordinations municipales-provinciales (en majorité sous domination provinciale). Il n'en demeure pas moins que les relations tripartites sont possibles et qu'elles sont, dans certains domaines, essentielles. La coopération peut produire des politiques efficaces. Celles-ci sont facilitées lorsque les fonctionnaires supérieurs procèdent à des consultations adéquates, qu'on peut compter sur un fort leadership au plan municipal et que les relations sont saines entre les politiciens des divers niveaux.

\section{- CONCLUSION}

Au cours des dernières années au Canada, on constate une certaine pression vers la décentralisation ainsi que de fortes réclamations des municipalités désireuses de jouer un rôle plus important, en particulier dans le cas des plus grandes villes, au sein du rapport de force de la gouvernance canadienne. Des analystes convaincants prétendent que les forces mondiales et les impératifs de la concurrence économique rendent cette évolution inévitable (Courchene, 2007). On constate un mouvement dans cette direction. La plupart des gouvernements provinciaux ont augmenté la portée de l'autonomie de " leurs » municipalités. Plus frappant, le Nouveau pacte pour les villes et les collectivités (2004-2006) visait à augmenter les ressources des administrations locales et à cimenter le partenariat fédéral-municipal dans l'élaboration des politiques au sein des villes. Le Nouveau pacte a été jeté aux oubliettes sans coup férir par le gouvernement conservateur.

Cependant, les relations municipales-provinciales-fédérales demeurent très importantes dans l'élaboration des politiques canadiennes, en particulier sous la forme de bilatéralisme ${ }^{8}$. Dans toutes ces relations, bilatérales ou multilatérales, les administrations municipales ont tendance à recevoir des politiques. Elles sont sujettes aux initiatives prises par les gouvernements provinciaux, dans un contexte minimal de consultations, dans une grande variété de domaines dont nous n'avons pas fait mention dans cet article (l'aménagement du territoire, les routes, la santé publique, les bibliothèques, la police, les codes du bâtiment, et plus encore). Les administrations municipales doivent également réagir aux initiatives politiques unilatérales du gouvernement fédéral dans des domaines tels que les immeubles fédéraux et les infrastructures. Dans de nombreuses relations intergouvernementales, les

8 Cette relation pourrait être qualifiée de « bilatéralisme en série » (Miller et Smart, 2012). Alors qu'il existe des relations trilatérales - par exemple lorsque des autorités municipales et provinciales font des pressions collectivement sur Ottawa à propos d'une propriété fédérale -, il existe d'autres cas où des ententes fédérales-provinciales sont suivies de négociations provinciales-municipales portant sur l'implantation de politiques. Dans d'autres cas encore, il faut le souligner, le bilatéralisme implique des négociations entre les gouvernements provinciaux et fédéraux d'une part, et les forces sociales locales de l'autre, tels les organismes d'établissement des immigrants, dotés de participations limitées des fonctionnaires municipaux. 
municipalités ne disposent pas des ressources et des compétences pour créer un impact politique significatif.

Nous émettons cependant l'opinion que les municipalités peuvent jouer un rôle plus grand et qu'elles peuvent poursuivre leurs objectifs avec succès dans l'arène intergouvernementale. Tout dépend de leur orientation. Il est possible de classer les administrations en deux catégories, les " minimalistes " et les " complètes » (Young, 2012, p. 16-17). Les administrations du premier type se concentrent sur la provision de services standards et tentent de les livrer efficacement de manière à garder le niveau des taxes et des frais le plus bas possible. Mais d'autres municipalités sont prêtes à aller plus loin que leur rôle traditionnel de fournisseurs de services pour prendre en charge des activités situées au-delà de ce qui leur est alloué par les gouvernements provinciaux. Elles sont prêtes à l'action et font preuve d'une nouvelle pleine maturité (Siegel et Tindal, 2006). Elles ne fonctionnent pas comme des unités administratives du gouvernement provincial, mais deviennent les moyens par lesquels les communautés formulent des objectifs communs et agissent en fonction de leur intérêt collectif ${ }^{9}$. Les administrations municipales dotées d'une orientation complète sont prêtes à prendre les devants dans la prise en charge des problèmes vécus sur leur territoire et, de cette façon, à engager les autres ordres de gouvernement. Pour que ces entreprises soient couronnées de succès, une fondation solide basée sur l'appui du public, exprimé de manière démocratique, est d'un secours considérable (Young, 2009b, p. 497-498).

Dans notre étude, nous avons révélé l'existence de certaines administrations locales prêtes à faire la promotion de leur image par le truchement de grands événements sportifs qui soutiraient du financement des autres paliers de gouvernement. D'autres ont employé le Programme des candidats des provinces de manière à attirer de nouveaux immigrants et ont entrepris de lancer des mesures originales afin de les conserver. Les Villes d'Edmonton et de Calgary n'ont aucune responsabilité envers les Autochtones en milieu urbain, mais elles s'engagent dans certaines élaborations de programmes et publient depuis longtemps un bottin des services offerts aux Autochtones par les autres gouvernements et les organismes de services locaux. Certaines municipalités coopèrent efficacement avec leurs voisins dans la planification des urgences. Des plans cohérents appuyés par un consensus local ont permis d'atteindre le succès dans le réaménagement de propriétés fédérales, ainsi que dans certains programmes d'infrastructure. De nombreux facteurs rendent certaines administrations municipales plus efficaces que d'autres (la taille, l'importance au plan électoral, les ressources, etc.), mais il est clair que celles jouissant d'une orientation complète ont plus de chances de viser le succès dans les arènes de la gouvernance multiniveau.

9 Cette distinction séparant la fourniture de services et l'action politique collective est présente d'un bout à l'autre d'un texte portant sur les administrations locales canadiennes (Tindal et Tindal, 2009). 


\section{BIBLIOGRAPHIE}

Agranoff, R. et M. McGuire (2003). Collaborative Public Management: New Strategies for Local Governments, Washington, Georgetown University Press.

Anderson, C. (2006). "Economic Voting and Multilevel Governance: A Comparative Individual-Level Analysis ", American Journal of Political Science, vol. 50, n² 2, p. 449-463.

Andrew, C., K. A. Graham et S. Phillips (dir.) (2002). Urban Affairs: Back on the Policy Agenda, Montréal et Kingston, McGill-Queen's University Press.

Berdahl, L. (2006). " The Federal Urban Role and Federal-Municipal Relations ", dans R. Young et C. Leuprecht (dir.), Canada: The State of the Federation 2004 - MunicipalFederal-Provincial Relations in Canada, Montréal et Kingston, McGill-Queens University Press for the Institute of Intergovernmental Relations, School of Policy Studies, Queen's University, p. 25-43.

Bherer, L. et P. Hamel (2012). "Overcoming Adversity, or Public Action in the Face of New Urban Problems: The Example of Montreal ", dans M. Horak et R. Young (dir.), Sites of Governance: Multilevel Governance and Policy Making in Canada's Big Cities, Montréal et Kingston, McGill-Queen's University Press, p. 104-135.

Bradford, N. (2007). Whither the Federal Urban Agenda? A New Deal in Transition, Canadian Policy Research Networks, Family Network, Research Report F/65.

Breton, A. (1987). "Towards a Theory of Competitive Federalism ", European Journal of Political Economy, vol. 3, n 1-2, p. 263-329.

Carroll, B. W. et K. A. H. Graham (dir.) (2009). Administration publique du Canada, numéro spécial sur le fédéralisme, la politique publique et les municipalités, vol. $52, n^{\circ} 3$.

Chenier, J. A. (2009). "The Evolving Role of the Federation of Canadian Municipalities ", Canadian Public Administration, vol. 52, n 3, p. 395-416.

Courchene, T. J. (2007). "Global Futures for Canada's Global Cities ", Policy Matters, vol. $8, n^{\circ} 2$.

Esselment, A. (2011). " Birds of a Feather? The Role of Partisanship in the 2003 Ontario Liberal Transition ", Canadian Public Administration, vol. 54, n 4, p. 465-486.

Esselment, A. (2010). "Fighting Elections: Cross-Level Political Party Integration in Canada ", Canadian Journal of Political Science, vol. 43, n 4, p. 871-892.

Gagnon, A.-G. (dir.) (2009). Contemporary Canadian Federalism: Foundations, Traditions, Institutions, Toronto, University of Toronto Press.

Garcea, J. et E. C. LeSage Jr. (dir.) (2005). Municipal Reform in Canada: Reconfiguration, ReEmpowerment, and Rebalancing, Toronto, Oxford University Press.

Grieve, M. et L. Turnbull (à paraître). "Emergency Planning in Nova Scotia ", dans D. Henstra (dir.), Multilevel Governance and Emergency Planning in Canadian Municipalities, Montréal et Kingston, McGill-Queen's University Press.

Haddow, R. (2011). " Multilevel Governance and Immigration Policy in Nova Scotia ", dans E. Tolley et R. Young (dir.), Immigrant Settlement Policy in Canadian Municipalities, Montréal et Kingston, McGill-Queen's University Press, p. 192-240.

Harper, S. (2010), Speech to the Federation of Canadian Municipalities, Toronto, 28 mai, Author's notes. 
Harvey, J. et R. Young (dir.) (2012). Multilevel Governance and Image-Building in Canadian Municipalities, Montréal et Kingston, McGill-Queen's University Press.

Henstra, D. (dir.) (à paraître). Multilevel Governance and Emergency Planning in Canadian Municipalities, Montréal et Kingston, McGill-Queen's University Press.

Hooghe, L. et G. Marks (2003). « Unraveling the Central State, but How? Types of Multilevel Governance ", American Political Science Review, vol. 97, n² 2, p. 233-243.

Horak, M. (2012a). « Multilevel Governance in Toronto: Success and Failure in Canada's Largest City ", dans M. Horak et R. Young (dir.), Sites of Governance: Multilevel Governance and Policy Making in Canada's Big Cities, Montréal et Kingston, McGillQueen's University Press, p. 228-262.

Horak, M. (2012b). "Conclusion: Understanding Multilevel Governance in Canada's Cities ", dans M. Horak et R. Young (dir.), Sites of Governance: Multilevel Governance and Policy Making in Canada's Big Cities, Montréal et Kingston, McGill-Queen's University Press, p. 339-370.

Horak, M. et R. Young (dir.) (2012). Sites of Governance: Multilevel Governance and Policy Making in Canada's Big Cities, Montréal et Kingston, McGill-Queen's University Press.

Hutton, T. (2012). « Multilevel Governance and Urban Development: A Vancouver Case Study ", dans M. Horak et R. Young (dir.), Sites of Governance: Multilevel Governance and Policy Making in Canada's Big Cities, Montréal et Kingston, McGill-Queen's University Press, p. 263-298.

Ircha, M. C. et R. Young (dir.) (à paraître). Multilevel Governance and Federal Property in Canadian Municipalities, Montréal et Kingston, McGill-Queen's University Press.

Lazar, H. et C. Leuprecht (dir.) (2007). Spheres of Governance: Comparative Studies of Cities in Multilevel Governance Systems, Montréal et Kingston, McGill-Queen's University Press for the Institute of Intergovernmental Relations, School of Policy Studies, Queen's University.

Leo, C. (2006). " Deep Federalism: Respecting Community Difference in National Policy ", Canadian Journal of Political Science, vol. 39, n³, p. 481-506.

Leuprecht, C. et H. Lazar (2007). " From Multilevel to "Multi-order" Governance? ", dans H. Lazar et C. Leuprecht (dir.), Spheres of Governance: Comparative Studies of Cities in Multilevel Governance Systems, Montréal et Kingston, McGill-Queen's University Press for the Institute of Intergovernmental Relations, School of Policy Studies, Queen's University, p. 1-21.

Miller, B. et A. Smart (2012). " Ascending the Main Stage?: Calgary in the Multilevel Governance Drama ", dans M. Horak et R. Young (dir.), Sites of Governance: Multilevel Governance and Policy Making in Canada's Big Cities, Montréal et Kingston, McGillQueen's University Press, p. 26-52.

OCDE (2012). Fiscal Decentralization Database, www.oecd.org/document/32/0,3746, en_2649_35929024_47467040_1_1_1_1,00.html\#SEC_B_2 (page consultée le 17 juin 2012).

Papadopoulos, Y. (2010). "Accountability and Multi-level Governance: More Accountability, Less Democracy? », West European Politics, vol. 33, n 5, p. 1030-1049.

Peters, E. J. (dir.) (2011). Urban Aboriginal Policy Making in Canadian Municipalities, Montréal et Kingston, McGill-Queen's University Press. 
Pierre, J. et B. G. Peters (2005). Governing Complex Societies: Trajectories and Scenarios, New York, Palgrave Macmillan.

Sancton, A. et R. Young (dir.) (2009). Foundations of Governance: Municipal Government in Canada's Provinces, Toronto, University of Toronto Press for the Institute of Public Administration of Canada.

Sanderson, C. et P. Filion (à paraître). " The Development of the Toronto Waterfront: Federal Presence, Institutional Complexity and Planning Outcomes ", dans M. Ircha et R. Young (dir.), Multilevel Governance and Federal Property in Canadian Municipalities, Montréal et Kingston, McGill-Queen's University Press.

Siegel, D. et C. R. Tindal (2006). " Changing the Municipal Culture: From Comfortable Subordination to Assertive Maturity ", Municipal World, partie 1, mars, p. 37-40 et partie 2, avril, p. 13-17.

Simeon, R. (2002). Political Science and Federalism: Seven Decades of Scholarly Engagement, 2000 Kenneth R. MacGregor Lecture, Kingston, Institute of Intergovernmental Relations, Queen's University.

Tindal, C. R. et S. N. Tindal (2009). Local Government in Canada, $7^{\mathrm{e}}$ éd., Toronto, Nelson Education

Tolley, E. et R. Young (dir.) (2011). Immigrant Settlement Policy in Canadian Municipalities, Montréal et Kingston, McGill-Queen's University Press.

Young, R. (2012). "Introduction: Multilevel Governance and Its Central Research Questions in Canadian Cities ", dans M. Horak et R. Young (dir.), Sites of Governance: Multilevel Governance and Policy Making in Canada's Big Cities, Montréal et Kingston, McGill-Queen's University Press, p. 3-25.

Young, R. (2011). "The Federal Role in Canada's Cities: The Pendulum Swings Again ", dans T. Courchene et autres (dir.), The Federal Idea: Essays in Honour of Ronald L. Watts, Montréal et Kingston, McGill-Queen's University Press et The Institute of Intergovernmental Relations, p. 313-322.

Young, R. (2009a). "Canada ", dans N. Steytler (dir.), Local Government and Metropolitan Regions in Federal Systems, A Global Dialogue on Federalism Volume VI, Senior Editor John Kincaid, Montréal et Kingston, McGill-Queen's University Press for the Forum of Federations and the International Association of Centers for Federal Studies, p. 106-135.

Young, R. (2009b). "Conclusion ", dans A. Sancton et R. Young (dir.), Foundations of Governance: Municipal Government in Canada's Provinces, Toronto. University of Toronto Press for the Institute of Public Administration of Canada, p. 487-499.

Young, R. (2006). " Open Federalism and Canadian Municipalities ", dans K. Banting et autres, Open Federalism: Interpretations, Significance, Kingston, Institute of Intergovernmental Relations, p. 7-24.

Young, R. et C. Leuprecht (dir.) (2006). Canada: The State of the Federation 2004 - MunicipalFederal-Provincial Relations in Canada, Montréal et Kingston, McGill-Queens University Press for the Institute of Intergovernmental Relations, School of Policy Studies, Queen's University. 


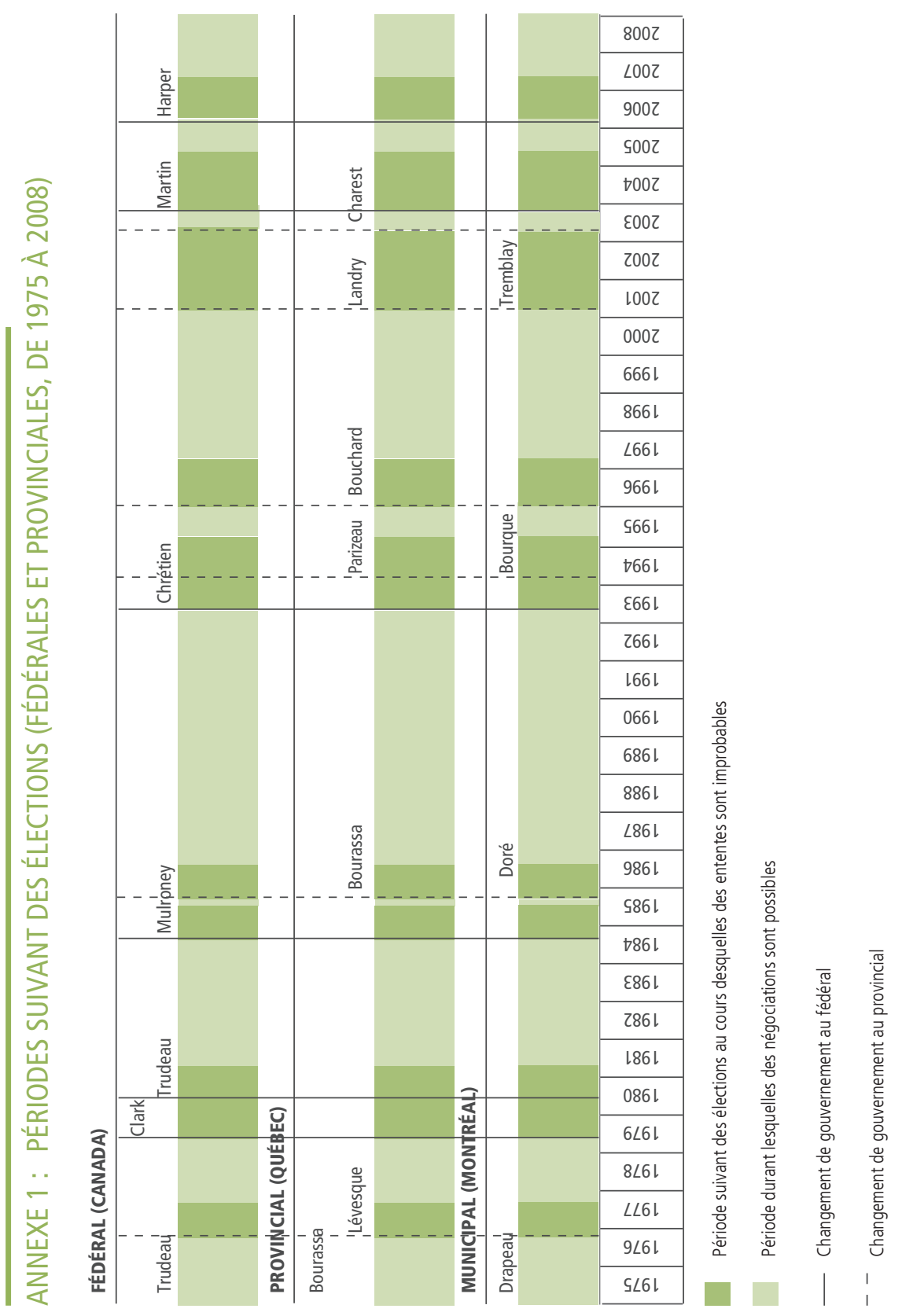




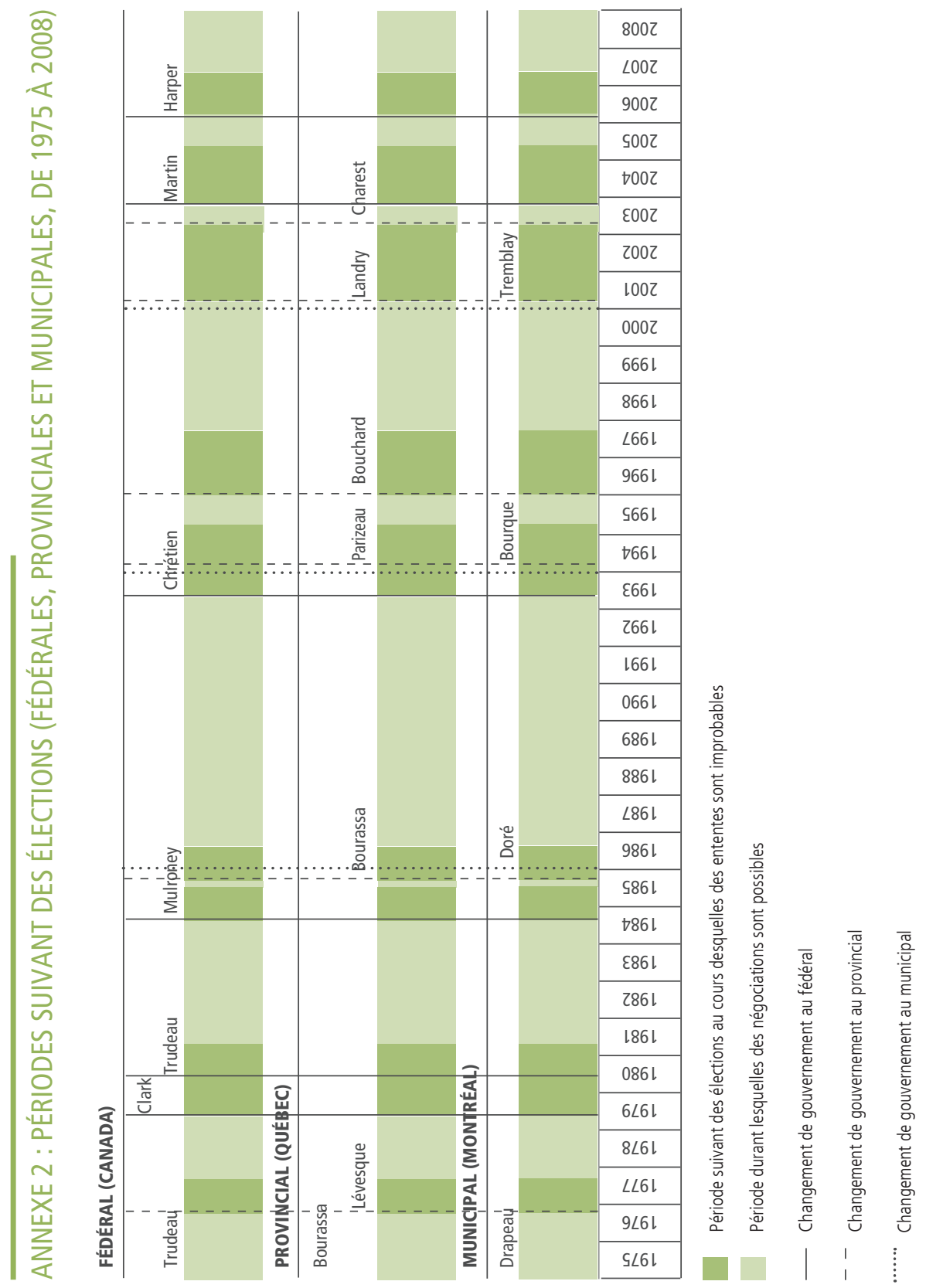

